\title{
Performance Assessment of a Single-Layer Metasurface Resonator for 3 T MRI
}

\author{
Endri Stoja, Dennis Philipp, Simon Konstandin, Diego Betancourt, Robin Niklas Wilke, \\ Reiner Umathum, Jürgen Jenne, Thomas Bertuch, and Matthias Günther
}

\begin{abstract}
Metamaterials, in particular 2D metasurfaces, offer great potential to advance measurement efficiency in magnetic resonance imaging (MRI) and to overcome some limitations due to physiological restrictions that hamper technically feasible developments. In particular, the signal-to-noise ratio in MRI can be improved significantly in many applications. One major drawback, however, of MRI-compatible metamaterials is their usually bulky structure, which limits possible applications and does not allow for flexible or conformally fitted metamaterial layers. This problem can be overcome by flat stripe-shaped resonator unit cells in linear metasurfaces. Such unit cells need to be coupled capacitively, which implies multilayer designs. Here, we take the next step and investigate the use of interdigital capacitors to electrically elongate the wires composing a single-layer metasurface resonator. A comprehensive study of the fundamental mode, which is of particular interest for MRI, is presented. To that end, the on-bench performance of three prototypes are compared, two of which are designed with the help of structural parallel-plate capacitors on two layers, and the last using the interdigital, single-layer approach. Although the adoption of interdigital capacitors simplifies prototyping, we observe that the quality factor drops
\end{abstract}

Manuscript received 28 December 2021.

Endri Stoja, Diego Betancourt, and Thomas Bertuch are with Antenna Technology and EM Modelling, Fraunhofer FHR, Fraunhoferstrasse 20, 53343 Wachtberg, Germany; e-mail: endri. stoja@fhr.fraunhofer.de, diego.betancourt@fhr.fraunhofer.de, thomas.bertuch@fhr.fraunhofer.de.

Dennis Philipp is with MR Physics, Fraunhofer MEVIS, Maxvon-Laue-Strasse 2, 28359 Bremen, Germany; and the University of Bremen, Otto-Hahn-Allee 1, 28359 Bremen, Germany; e-mail: dennis.philipp@mevis.fraunhofer.de.

Simon Konstandin and Robin Niklas Wilke are with MR Physics, Fraunhofer MEVIS, Max-von-Laue-Strasse 2, 28359 Bremen, Germany; e-mail: simon.konstandin@mevis.fraunhofer. de, robin.wilke@mevis.fraunhofer.de.

Reiner Umathum is with MR Physics, Fraunhofer MEVIS, Maxvon-Laue-Strasse 2, 28359 Bremen, Germany; and the Division of Medical Physics in Radiology, German Cancer Research Center DKFZ, Im Neuenheimer Feld 280, 69120 Heidelberg, Germany; email: reiner.umathum@mevis.fraunhofer.de.

Jürgen Jenne is with MR Physics, Fraunhofer MEVIS, Maxvon-Laue-Strasse 2, 28359 Bremen, Germany; and Clinical Cooperation Unit Molecular Radiooncology, German Cancer Research Center DKFZ, Im Neuenheimer Feld 280, 69120 Heidelberg, Germany; e-mail: juergen.jenne@mevis.fraunhofer.de.

Matthias Günther is with MR Physics, Fraunhofer MEVIS, Max-von-Laue-Strasse 2, 28359 Bremen, Germany; and MRImaging and Spectroscopy, Faculty 01, University of Bremen, Otto-Hahn-Allee 1, 28359 Bremen, Germany; e-mail: matthias. guenther@mevis.fraunhofer.de. significantly. However, MRI experiments show promising results, leading to significant enhancement of the signal-to-noise ratio in the region of interest. Ultimately, single-layer structures open the possibility of flexible and conformal designs to optimally adapt to the patient's body in MRI.

\section{Introduction}

Although magnetic resonance imaging (MRI) has been used and improved for more than five decades as the most versatile medical imaging modality, there still is a lot of potential to exploit its full capabilities. Mainly physiological restrictions hamper some physically feasible developments. Thus, it becomes challenging to innovate and advance the field in terms of, for example, imaging efficiency. Electromagnetic metamaterials are a promising solution in this respect, since they allow substantial enhancement of the signal-to-noise ratio (SNR) [1, 2], which benefits resolution and scanning time. A major drawback is the usually bulky design of the metamaterial structures, putting patient-specific and conformal metasurfaces for versatile applications beyond reach. However, the development of thin (submillimeter) dual-layer metasurfaces [2] overcomes these problems and is the first step toward even more advanced applications. Moreover, smart self-detuning strategies in the transmit phase of MRI (TX) allow patient safety to be taken care of at all times $[2,3]$. The combination of both thin metasurfaces and inherent self-detuning allows for the most advanced metasurfaces for MRI to date.

Here we advance the development by proceeding toward single-layer metasurfaces, which can be manufactured in a single step by a state-of-the-art laser etching process. These metasurfaces make use of interdigital capacitors [4], which replace the parallelplate two-layer structures in [2].

The metasurface is composed of 14 wire-resonator shaped unit cells (copper stripes) in a linear array, which is etched on RO4003 substrate $(0.508 \mathrm{~mm}$ thickness) with the dimensions as indicated in Figure 1.

We report on the design of the interdigital capacitors and the characterization of prototypes via on-bench and MRI performance tests. To evaluate the performance of the single-layer design for existing structures, a comparison to the parallel-plate (PP) embodiment is presented as well.

\section{Interdigital Capacitor Design}

The interdigital capacitors (IDs) shown in Figure 1 are composed of 28 copper "fingers" of about $5 \mathrm{~mm}$ 


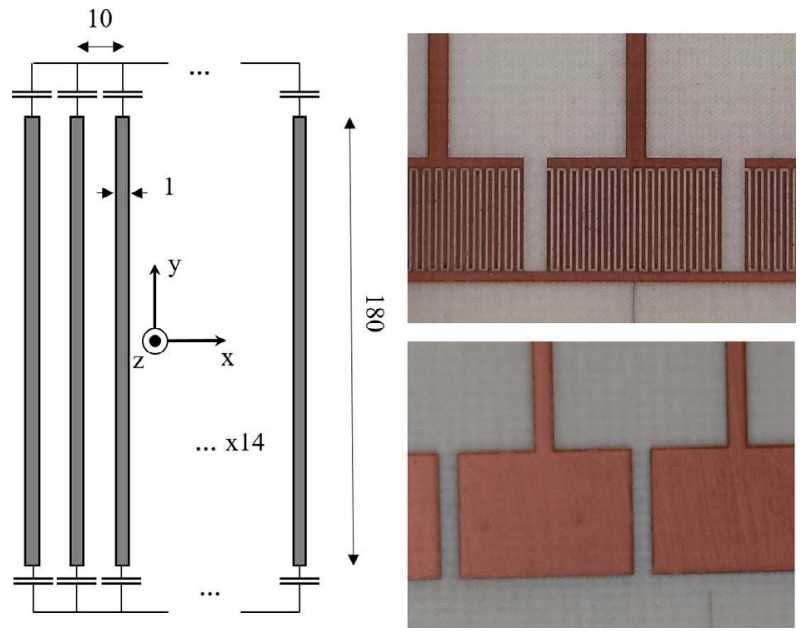

Figure 1. (Left) Schematic representation of the enhancement plate (all lengths in mm); (right) details of the fabricated capacitors - (top) ID and (bottom) PP types.

length and $160 \mu \mathrm{m}$ width each. The gap between them is also $160 \mu \mathrm{m}$. The total effective area occupied by each capacitor is $6.25 \mathrm{~mm} \times 8.8 \mathrm{~mm}$, which is almost the same as that of the rectangular patches in the PP case (6 $\mathrm{mm} \times 9 \mathrm{~mm})$.

For the design, eigenmode simulations performed in CST Microwave Studio 2019 were adopted for optimization after a first estimation of the required capacitance values was achieved using a lumped-element circuit model [5], exploiting the alternative view of the metasurface as an open low-pass birdcage coil and subsequent interdigital capacitor geometry derivation from the analytical formulas in [4]. Laser etching provides rapid prototyping capabilities. Note that for the PP-cap prototype, a ground stripe on the back layer (opposing the patches in Figure 1) needs to be added.

\section{On-Bench Mode Imaging and Comparison}

The resonance frequency and the quality factor of the ground mode were measured on-bench with the $S_{21}$ dual-loop technique [6]. The ID prototype has a quality factor of 130, almost half of the PP version's 250, and the respective resonant frequencies are $128.6 \mathrm{MHz}$ and 127.4 MHz. These are intentionally higher than the target frequency, to compensate for phantom loading in MRI scans. A compression of the higher-order odd mode frequencies is observed in the latter case. To image the modes, a broadband tuned coil of $30 \mathrm{~cm}$ diameter excites the plate positioned $4 \mathrm{~cm}$ above it, and a $10 \mathrm{~mm}$ sniffer loop is used to locally sample $S_{21}$ across a plane $1 \mathrm{~cm}$ from the plate (Figure 2).

Scans across the symmetry planes of the setup were performed as well. For the mode of interest, the maximum $\mathrm{S}_{21}$ is $1.2 \mathrm{~dB}$ lower than in the ID case, and the enhancement with respect to penetration depth is similar (Figure $3 \mathrm{~b}-1$ ). With regard to homogeneity perpendicular to the stripes, the same behavior was observed.

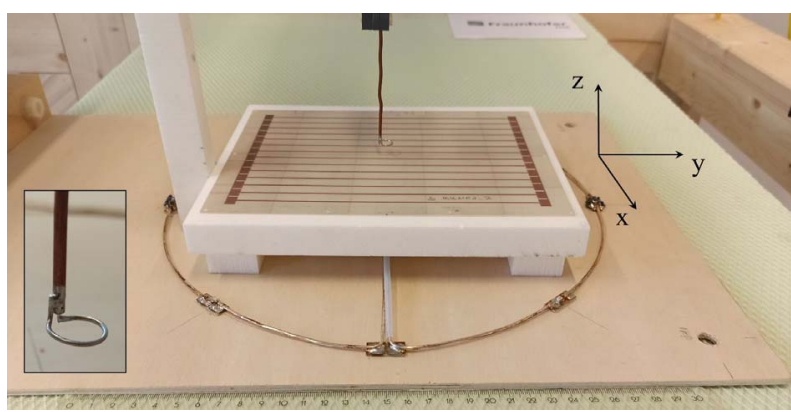

Figure 2. Measurement setup for mode characterization.

\section{MRI Experiments}

To use metasurfaces in an MRI environment, the introduction of an (external) inductively coupled loop that senses the field strength and thus triggers the detuning of the device via a limiting diode pair during TX is advisable [7]. In this way, we can protect the patient and potential electronics from the impact of high power in small volumes (hot spots). This approach requires modification of the capacitor values in the primary resonator in an iterative design cycle. This is achieved in both the PP and ID cases by varying the length of the patches and of the fingers, respectively. Moreover, the addition of a trimmer capacitor (Figure 4t-1) allows for on-bench fine-tuning at different loading conditions and eases the requirements on prototype manufacturing accuracy.
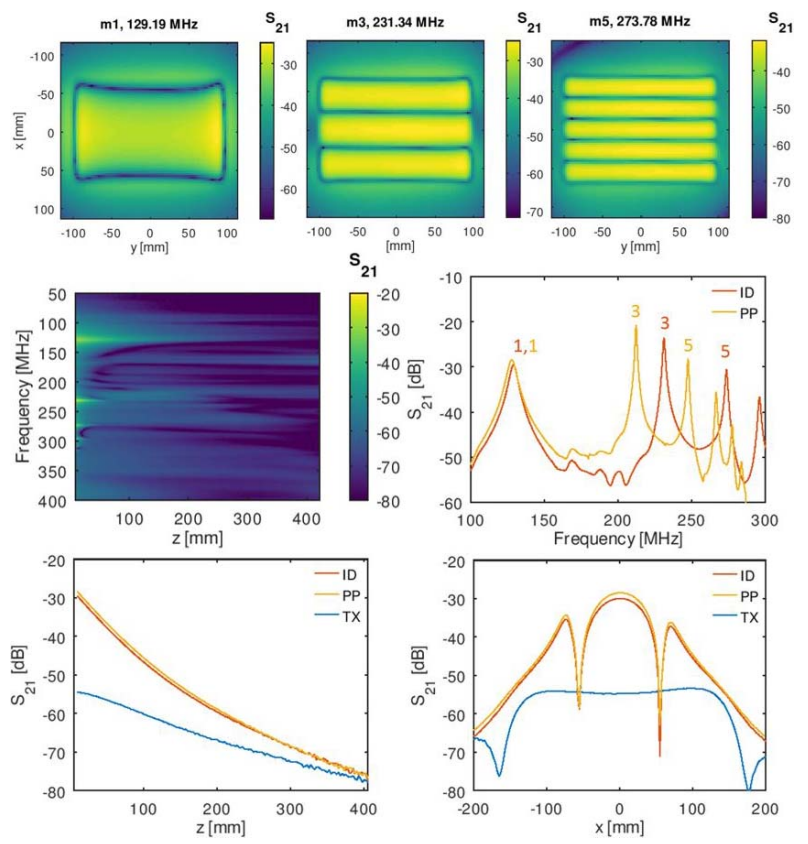

Figure 3. (Top) $\mathrm{S}_{21}$ maps of first three odd modes of the ID plate 10 $\mathrm{mm}$ above the surface; (middle left) $\mathrm{S}_{21}(\mathrm{z}$ ), allowing observation of the different modes and their penetration depths; (middle right) $S_{21}$ at the center of the metasurfaces, with indicated mode numbers; (bottom left) $S_{21}(z)$ for mode 1; (bottom right) $S_{21}$ perpendicular to the stripes at $10 \mathrm{~mm}$ height. 

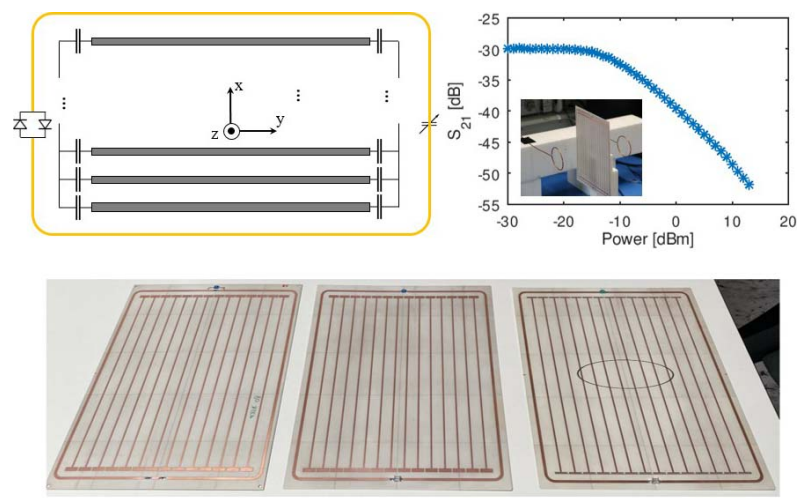

PP1

PP2

ID3

Figure 4. (Top left) Schematic illustration of the self-detuning resonator; (top right) on-bench verification of the self-detuning at increasing power by use of two untuned $60 \mathrm{~mm}$ diameter sniffers; (bottom) the three prototypes used for the comparison.

For the PP approach, we use two different prototypes for the comparison: PP1, making use of three affordable, off-the-shelf RF diodes (Skyworks SMV2020), and PP2, which adopts an MRI-specific limiting diode pair (Microsemi UMX9989AP). Likewise, the new ID prototype is denominated ID3, to distinguish it from the one considered in Section 3.

In the MRI experiment, performed with a $3 \mathrm{~T} \mathrm{MR}$ scanner (Magnetom Skyra, Siemens Healthineers), a cylindrical homogeneous phantom is used. The metasurface under investigation is positioned at the isocenter of the scanner's bore, with the wires perpendicular to the bore's axis at a distance of $10 \mathrm{~mm}$ from the nearest extremity of the phantom (which is also aligned with the bore). A 2D FLASH sequence was used, and we recorded magnitude and phase images with the following sequence parameters: $\mathrm{TR}=100 \mathrm{~ms}, \mathrm{TE}=5$ $\mathrm{ms}$, field of view $=128 \mathrm{~mm}$, matrix $=128$, eight slices with a thickness of $5 \mathrm{~mm}$ and slice distance factor of $200 \%$, and 10 measurements with flip angles between $0^{\circ}$ and $90^{\circ}$.

By using the scanner's built-in body coil in the receive phase, several axial slices were recorded, with slice 4 passing through the isocenter and showing the maximum SNR. Note that the body coil was used for TX throughout all experiments. Two different regions of interest were defined for SNR evaluation (Figure 5). The first one is close to the metasurface, whereas the second one is located at the specular extremity of the phantom. This allows us to discriminate the SNRenhancement capabilities of the metasurfaces at different distances from the structures.

To verify that all the prototypes detune efficiently during the experiment (in the TX phase), a nominal flipangle sweep was performed while observing the SNR behavior [2]. The results are presented in Figure 6. As can be observed, the maxima of the curves, at the socalled Ernst angle, do not shift when the metasurfaces are present. This indicates proper detuning at high incident power, and corresponding higher SNR values
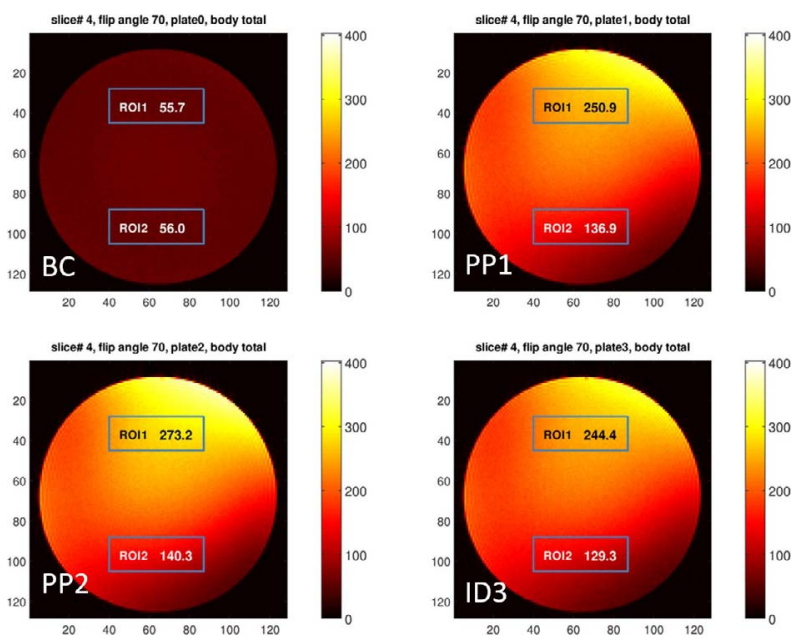

Figure 5. SNR maps of (top left) the scanner's body coil only and body coil + (top right) PP1, (bottom left) PP2, and (bottom right) ID3. The metasurface is located $10 \mathrm{~mm}$ above the phantom. Blue rectangles delimit the ROIs; numbers indicate the SNR evaluation in the ROI.

show that effective SNR enhancement occurs during the receive phase. For the ID3 case, we find an enhancement factor of about 5 compared to the reference measurement without any metasurface.

The SLC curve corresponds to the case when a 70 $\mathrm{mm}$ diameter circular surface loop coil positioned 10 $\mathrm{mm}$ on top of the phantom is used in the receive phase with no metasurface present. Although this may not be a fair comparison, due to the area mismatch between the metasurfaces and the surface loop coil [6], it can be shown that with regard to region of interest 1 , the

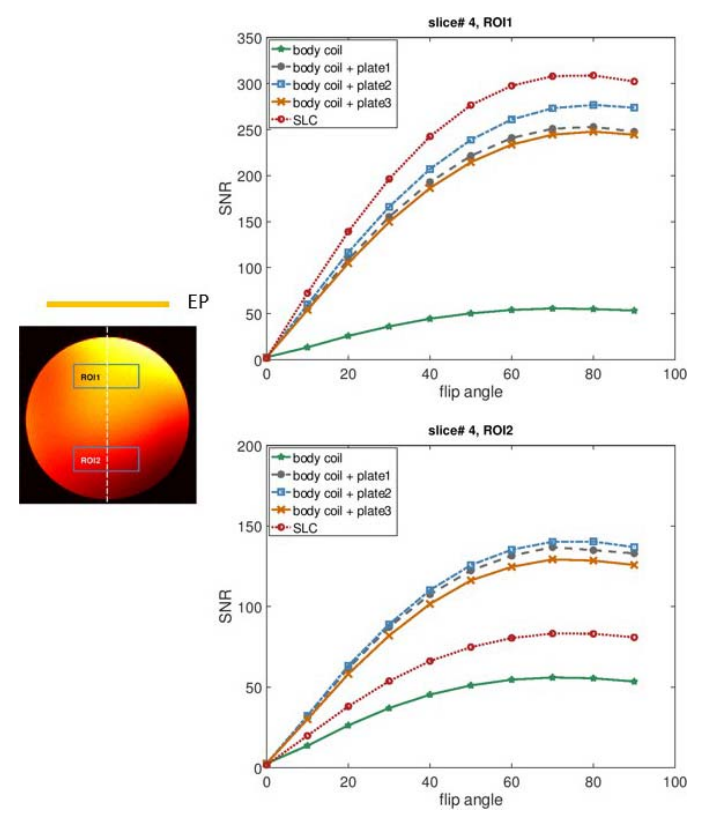

Figure 6. SNR vs. nominal flip angle for (top) ROI1 and (bottom) ROI2. (Left) The positioning of the metasurface (enhancement plate, EP) relative to the phantom. 
metasurfaces all perform at a similar level as that particular surface loop coil.

MRI measurements confirm the SNR enhancement from the single-layer metasurface, although to a smaller extent than in the PP case, due to the smaller quality factor of the core-resonator. The use of affordable RF diodes for self-detuning loops rather than the MRI-specific ones also slightly lowers the performance.

\section{Conclusions}

We have experimentally shown that single-layer metasurfaces allow spatial modulation of the near-field in MRI, leading to a significant SNR enhancement in comparison to imaging with RF coils only. This study paves the way for the design of new imaging coils with integrated metasurfaces, offering on-the-fly customizability. Flexible and conformal metasurfaces, as well as printable ones for patient-customized use, will be investigated in the future.

\section{Acknowledgment}

This work was supported by the Fraunhofer Internal Program (grant MAVO 142-600555).

\section{References}

1. A. P. Slobozhanyuk, A. N. Poddubny, A. J. E. Raaijmakers, C. A. T. van den Berg, A. V. Kozachenko, et al., "Enhancement of Magnetic Resonance Imaging With Metasurfaces," Advanced Materials, 28, 9, March 2016, pp. 1832-1838, doi: 10.1002/adma.201504270.

2. E. Stoja, S. Konstandin, D. Philipp, R. N. Wilke, D. Betancourt, et al., "Improving Magnetic Resonance Imaging With Smart and Thin Metasurfaces," Scientific Reports, 11, August 2021, p. 16179, doi: $10.1038 / \mathrm{s} 41598$ 021-95420-w.

3. X. Zhao, G. Duan, K. Wu, S. W. Anderson, and X. Zhang, "Intelligent Metamaterials Based on Nonlinearity for Magnetic Resonance Imaging," Advanced Materials, 31, 49, December 2019, p. 1905461, doi: 10.1002/adma. 201905461.

4. I. J. Bahl, Lumped Elements for RF and Microwave Circuits, Boston, Artech House, 2003.

5. J. Jin, Electromagnetic Analysis and Design in Magnetic Resonance Imaging, Boca Raton, FL, CRC Press, 1999.

6. J. T. Vaughan, and J. R. Griffiths (eds.), RF Coils for MRI, New York, John Wiley and Sons Ltd., 2012.

7. E. Stoja, D. Philipp, D. Betancourt, S. Konstandin, R. N. Wilke, et al., "Numerical Investigation of a Self-Detuning Signal Enhancement Metasurface for 3T MRI," 2020 XXXIIIrd General Assembly and Scientific Symposium of the International Union of Radio Science, Rome, Italy, August 29-September 5, 2020, doi: 10.23919/URSIGASS 49373.2020.9232401. 